Vocal Development with Two Cochlear Implants: Authors' accepted manuscript (AAM)

Vocal Development in Infants and Toddlers with Bilateral Cochlear Implants and Infants with Normal Hearing

Taina T. Välimaa ${ }^{1}$ and Sari M. Kunnari ${ }^{1}$, University of Oulu, Finland

Päivi Laukkanen-Nevala²

Oulu, Finland

David J. Ertmer ${ }^{3}$

Purdue University

Author Note

Taina T. Välimaa, ${ }^{1}$ Faculty of Humanities, Research Unit of Logopedics and Child Language Research Center, University of Oulu, Finland; Sari M. Kunnari, ${ }^{1}$ Faculty of Humanities, Research Unit of Logopedics and Child Language Research Center, University of Oulu, Finland; Päivi Laukkanen-Nevala, ${ }^{2}$ Oulu, Finland; David J. Ertmer, ${ }^{3}$ Professor Emeritus, Department of Speech, Language, and Hearing Sciences, Purdue University, Indiana, USA.

This study was supported by the Academy of Finland.

Correspondence concerning this article should be addressed to Taina T. Välimaa, Faculty of Humanities, Research Unit of Logopedics and Child Language Research Center, P.O. Box 1000, 90014 UNIVERSITY OF OULU, Finland. E-mail: taina.valimaa@oulu.fi Conflict of Interest

The authors state that they have no relevant conflicts of interest. 
Vocal Development with Two Cochlear Implants: Authors' accepted manuscript (AAM)

\begin{abstract}
Purpose: The purpose of this study is to investigate the time-course of vocal development in infants and toddlers with bilateral cochlear implants (bilateral CI group) who are acquiring Finnish and to compare their progress to that of infants with normal hearing and typical development (TD group).

Method: A total of 5964 spontaneous utterances of 30 infants and toddlers (15 in both groups) were classified as either Precanonical vocalizations (PC), Basic Canonical Syllables (BCS) or Advanced Forms (AF) levels. Time-course of development and group differences were analyzed in a prospective longitudinal study during a time-course of one year: before implantation and 1, 3, 6, 9 and 12 months after CI activation for the bilateral CI group and at 6, 9 and 12 months of age for the TD group.
\end{abstract}

Results: The least mature PC vocalizations decreased and the BCS and the AF vocalizations increased for both the bilateral CI and the TD groups during the follow-up period of one year. The bilateral CI group produced a lower percentage of PC vocalizations ( $\eta P^{2}$ effect size 0.35 ) and a higher percentage of $\mathrm{BCS}\left(\eta P^{2}\right.$ effect size 0.16$)$ and $\mathrm{AF}$ vocalizations ( $\eta P^{2}$ effect size 0.24) than the TD group.

Conclusions: The findings of this study showed that vocal development of infants and toddlers with early-identified profound hearing loss is delayed before CI activation. Findings also showed that infants and toddlers with bilateral CIs make rapid advancements in vocal development after implantation compared to infants with TD. However, their vocal development seems to remain delayed at least during the first year of bilateral CI use as compared to the well-documented milestones of infants and toddlers with TD. Information about the vocal development time-course following bilateral CI activation helps parents recognize progress in auditory-guided speech development before the emergence and the use of spoken words in communication. 
Vocal Development with Two Cochlear Implants: Authors' accepted manuscript (AAM)

Vocal Development in Infants and Toddlers with Bilateral Cochlear Implants and Infants with Normal Hearing

Prelinguistic vocal development is the gradual acquisition of mature speech patterns during the first two years of life characterized by the emergence of various kinds of vocalization types and overlapping levels of development. This process begins soon after birth with non-speech-like vocalizations such as grunts and cooing. Precanonical vowel-like sounds and laughter begin to emerge in the vocalizations usually at 4-6 months. As infants mature further, they progress to speech-like consonant-vowel (CV) combinations (canonical syllables) and utterances containing complex syllable shapes and an increasing array of vowels and consonants (Koopmans-van Beinum \& van der Stelt, 1986; Oller, 2000 p. 179180; Nathani, Ertmer, \& Stark, 2006; Roug, Landberg, \& Lundberg, 1989; Stark, 1980; see Vihman, 1996 for review). The onset of babbling at 6-10 months is one of the most important milestones in vocal development, even though the earlier stages also demonstrate the developing speech capacity (Oller, 2000, p. 179-180; Oller, Eilers, Neal, \& Schwartz, 1999; Vihman, 1996, p. 109). Vocalizations from each prelinguistic level continue to be observed as first words are produced by toddlers with typical development (TD). Because babbling and early meaningful speech show clear continuity, vocal development serves as a foundation for phonological development in children (Oller \& Eilers, 1988; Stoel-Gammon, 1985; Vihman, Macken, Miller, Simmons, \& Miller, 1985). The presence of severe-to-profound hearing loss (HL) during infancy can impede and limit progress in this vital developmental phenomenon. The limitations include, for example, delays in the establishment and amount of canonical syllables (Oller \& Eilers, 1988; Oller, 2000), and limited variety of syllable shapes (Moeller et al., 2007). These developmental delays may have a negative impact on the acquisition of spoken language skills (Davis, Morrison, von Hapsburg, \& Warner Czyz, 2005; Eilers \& 
Vocal Development with Two Cochlear Implants: Authors' accepted manuscript (AAM)

Oller, 1994; Moeller et al., 2007, Oller \& Eilers, 1988; Schauwers, Gillis, \& Govaerts, 2008; Stoel-Gammon \& Otomo, 1986).

Children who have no secondary disabilities and receive unilateral CIs within the first three years of life often make relatively rapid progress in vocal development compared to younger, TD children (Ertmer \& Jung, 2012; Ertmer \& Mellon, 2001; Ertmer, Young, \& Nathani, 2007; Fagan, 2015; Moreno-Torres, 2014; Schauwers, Gillis, Daemers, de Beukelaer, \& Govaerts, 2004). This outcome is encouraging in that it suggests that children with profound HL are building a foundation for phonological development through exposure to auditory models and auditory feedback. Recently, bilateral implantation has become a widespread practice. Bilateral CI use has led to better sound localization and speech perception ability in background noise than previously reported for children with unilateral CI (see Johnston, Durieux-Smith, Angus, O’Connor, \& Fitzpatrick, 2009 for a review). Some findings indicate that infants and toddlers with bilateral CIs are more likely to use vocalization to communicate, and to use audition when interacting vocally with an adult compared to infants and toddlers with unilateral CIs (Tait et al., 2010). It seems reasonable to hypothesize that greater benefits might be gained also in the acquisition of mature speech patterns as children receive CIs at even younger ages and both ears are implanted. However, very little is known about the initial phases of speech development in infants who are identified through newborn hearing screening and receive bilateral CIs close to their first birthdays. Additionally, none of the previous studies have reported vocal development of infants and toddlers acquiring Finnish. The current study attempted to overcome these deficits in knowledge by investigating vocal development in a group of infants and toddlers who received bilateral CIs at the mean age of 13 months and who were acquiring Finnish. Their progress in vocal development was compared to a control group of infants with $\mathrm{NH}$.

\section{Vocal development in infants and toddlers with cochlear implants}


Vocal Development with Two Cochlear Implants: Authors' accepted manuscript (AAM)

Infants and toddlers who have undergone unilateral cochlear implantation during the first three years of life have made significant progress in vocal development after device activation compared to TD infants and with similar experience of robust hearing (auditory access to speech at conversational intensity levels; Ertmer \& Jung, 2012; Ertmer, Jung, \& True Kloiber, 2013; Schauwers et al., 2004). Canonical babbling has been reported to start one to six months after implant activation compared to the emergence of this milestone at 6-10 months of age in TD infants (Ertmer \& Mellon, 2001; Ertmer \& Jung, 2012; Fagan, 2015; McCaffrey, Davis, MacNeilage, \& von Hapsburg, 1999; Moore \& Bass-Ringdahl, 2002; Schauwers et al., 2004). Clear advancements have been evident also in syllable shapes and phoneme inventories as well as volubility (Fagan, 2014; McCaffrey et al., 1999; Schauwers et al., 2008). Young CI recipients appear to be able to use audition to guide the production of canonical syllables and other aspects of phonological abilities soon after the activation of their devices.

Some studies have applied multi-level models to examine vocal development throughout the first two years of life. One such model is the Stark Assessment of Early Vocal Development-Revised (SAEVD-R; Nathani et al., 2006) that classifies infant vocalizations to five levels according to their development: 1) Reflexive Vocalizations, 2) Control of Phonation, 3) Expansion Phase, 4) Basic Canonical Syllables, and 5) Advanced Forms. Later on, Ertmer and Nathani Iyer (2010) developed a more consolidated version of the SAEVD-R that includes three levels instead of five: 1) Precanonical Vocalizations (PC; SAEVD-R levels 1-3), 2) Basic Canonical Syllables (BCS; SAEVD-R level 4), and 3) Advanced Forms (AF; SAEVD-R level 5).

Using the Consolidated Stark Assessment of Early Vocal Development-Revised (CSAEVD-R), Ertmer and Jung (2012) analyzed vocal development of 13 infants and toddlers with CIs at 3, 6, 9, and 12 months after CI activation and compared it to that of 
Vocal Development with Two Cochlear Implants: Authors' accepted manuscript (AAM)

infants with $\mathrm{NH}$ who had comparable amounts of robust hearing (i.e., at the chronological ages of 6, 9, and 12 months). For the CI group, the amount of robust hearing was equivalent with the months of CI use. The researchers found that young CI recipients produced significantly fewer PC vocalizations at six, nine, and 12 months post-activation than infants with $\mathrm{NH}$ at the chronological ages of six, nine, and 12 months. Thus, the relatively older children in the CI group required fewer months of robust hearing to decrease their productions of the least mature vocalizations measured by the CSAEVD-R. Similarly, the BCS level was established at $\geq 20 \%$ of the sample after three months of CI use (“establishment" criterion previously applied by Oller \& Eilers, 1988). For the TD group this level was not established until the age of 12 months. Lastly, the AF level was established after nine months of CI use, but not established and seldom observed for the TD group by 12 months of age. However, there are several limitations in applying the results of the Ertmer and Jung (2012) study to infants and toddlers who are currently bilaterally implanted following identification through newborn hearing screening.

In Ertmer and Jung's (2012) study, the mean age at CI activation ( $M=20$ months) was relatively high by today's standards. The children also showed a wide range of ages at activation (9-36 months), and most of the infants and toddlers had received a unilateral CI (10/13 participants). Three of the CI toddlers had bilateral CIs, but the percentages of vocalizations from each category at each interval were not significantly different for children with one versus two CIs. The authors also presented limited data on the vocal development levels before implant activation (only 2/13 participants). Thus, the lack of baseline information for the CI group makes it somewhat difficult to be confident of the children's actual stage of vocal development before implantation. Additionally, as the authors also discuss, two of the toddlers in their study had measurable residual hearing before implantation, which might have affected the time-course for vocal development. 
Vocal Development with Two Cochlear Implants: Authors' accepted manuscript (AAM)

The current study will attempt to overcome these limitations by using the CSAEVD-R (Nathani Iyer \& Ertmer, 2010) to track vocal development in children who represent today's standard for cochlear implantation: children implanted close to 12 months of age who received bilateral CIs. Because children in both the bilateral CI and the control group are acquiring Finnish, some of the phonemic and syllable shape features of Finnish will be compared to General American English in the next section.

\section{Characteristics of Finnish}

The Finnish consonant inventory consists of 13 phonemes (/p, t, d, k, n, m, y, l, j, v, r, s, $\mathrm{h} /$ ) and a vowel inventory of eight phonemes (/i, y, e, $\varnothing, \mathfrak{x}, \mathrm{u}, \mathrm{o}, \mathrm{a}, /$ ) (Kunnari \& SavinainenMakkonen, 2007; Suomi, Toivanen, \& Ylitalo, 2006). The maximum consonant inventory also contains /b, g, f, $\mathrm{f} /$, which may occur in loan words only (words originating from, e.g. Germanic languages). Finnish has phonologically contrastive lengths for both vowels and consonants. That is, the quantity of a sound (short vs. long) changes the meaning of the word (e.g., [kuka] 'who' vs. [kuk:a] 'flower', [tuli] 'fire' vs. [tu:li] 'wind'). All vowels and consonants (with the exception of /d, h, j, v/) can be long in a word-medial position. Finnish has 18 common diphthongs and about 50 word-medial heterosyllabic clusters (e.g., kan.sa 'people') but word-initial and word-final clusters are rare: word-initial clusters occur mostly in loan words and word-final clusters in interjections (e.g., poks 'pop'). Finnish also has 15 frequently occurring syllable shapes; about 58 percent of them are open and 42 percent are closed. CV is the most frequent syllable type, followed by CVC and CVV, the shortest possible syllable consists of a single vowel (e.g., o.vi 'door'), and the longest syllable contains four segments (e.g., pilk.ku 'dot') (Häkkinen, 1978; Suomi et al., 2006). The most frequent words in Finnish are bisyllabic, but words with three or more syllables are also common. 
Vocal Development with Two Cochlear Implants: Authors' accepted manuscript (AAM)

In contrast to Finnish, the General American English consonant inventory consists of 24

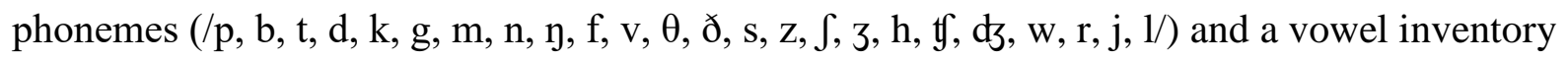

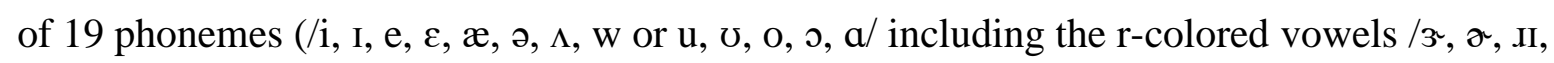
ع.I, JI, J., a./) (Bosma Smit, 2007). General American English has only three or four phonemic diphthongs, but many consonant clusters at both word-initial and word-final positions; in the final position clustered obstruents must agree in voicing. General American English also has a large inventory of syllable shapes: the shortest possible syllable consists of a single vowel (e.g., in the word 'I') like in Finnish, but very complex syllables such as CCCVCCC are also possible (e.g., in the word 'sprints'). General American English is very rich in monosyllables due to the possibility of singleton consonants and clusters at both the beginning and the end of the words. Some of these cross-linguistic differences may be apparent when the CSAEVD-R is used to study vocal development in young children acquiring Finnish.

\section{The current study}

In summary, prior research involving infants and toddlers with unilateral CI in heterogeneous age-at-implantation groups have shown relatively rapid progress in vocal development after device activation. It has not been determined whether the combination of younger age of implantation (i.e., close to 12 months) and bilateral CI use results in comparable timeframes for advancements in vocal development. There is also limited knowledge on typical patterns of vocal development in Finnish infants (Liukkonen \& Kunnari, 2012). Thus, the aims of the current study were: (1) to determine the time-course of vocal development for infants and toddlers with bilateral CIs who are acquiring Finnish, (2) to determine the time-course of vocal development for NH and TD infants who are acquiring Finnish, and (3) to compare the time-course of vocal development between these two groups. 
Vocal Development with Two Cochlear Implants: Authors' accepted manuscript (AAM)

The current study provides new information in several important ways. First, research on vocal development in infants and toddlers with CIs who are acquiring Finnish does not currently exist. Also, very little is known about typical patterns of vocal development in Finnish infants (Liukkonen \& Kunnari, 2012). Language-specific features in consonant production have been documented already at eight to ten months of age, which might be evident also in vocal development (Boysson-Bardies \& Vihman, 1991; Kern \& Davis, 2009; Levitt \& Aydelott Utman, 1992). Second, the infants in the current study were bilaterally implanted close to their first birthdays. They began to experience robust hearing (i.e., access to speech at conversational intensity levels; Ertmer \& Jung, 2012; Ertmer, Jung, \& True Kloiber, 2013) with the help of two CIs within an age range when the process of vocal development is typically happening. Many of the previous reports have included relatively older ages-at-implant, a wider range of ages and unilaterally implanted children. Thus, the current study will provide information on typical patterns of development as well as on the performance of early-diagnosed young bilateral CI recipients.

\section{Methods}

\section{Participants}

Two groups of infants and toddlers participated in this study. The first group was comprised of 15 infants (ten boys and five girls) with bilateral CIs received either simultaneously, or if sequentially $(n=3)$, with only a short interval between the first and the second implantation (range 4-10 months) (hereafter referred to as the bilateral CI group; see Table 1). The infants came from a nationwide prospective multi-center research project involving all the five university hospitals in Finland (i.e., Helsinki, Kuopio, Oulu, Tampere, and Turku). The Ethical Committee of the Northern-Savonia Hospital District, University Hospital of Kuopio, approved the study as ethically acceptable. The approval was verified in all the other University Hospitals according to their protocols. Written consent was received 
Vocal Development with Two Cochlear Implants: Authors' accepted manuscript (AAM)

from all the parents. The infants and toddlers in the bilateral CI group were diagnosed with congenital profound HL (WHO, 2015) through a combination of newborn hearing screening and Auditory Brainstem Responses or Auditory Steady-State Responses. Infants diagnosed with heart conditions, developmental brain disorders, chromosome anomalies or impairments in both hearing and vision were excluded. The infants' mean age at first HA fitting was 5.8 months $(S D=2.7$; range 3-14 months) and they all had at least a three-month HA trial before implantation. The infants' mean age at first CI activation was 13.3 months $(S D=3.2$; range 10-22 months).

All infants and toddlers in the bilateral CI group had hearing parents. The native language of both parents was Finnish for all but one (for whom Finnish was the native language of the mother). The main communication mode used by the parents with their infants and toddlers was speech, supported with some signs (and Cued Speech, $n=2$ ) (see Table 1). According to clinical practices in Finland, the families received basic instruction in signing after HL diagnosis, if desired. Some parents also voluntarily participated in a course on Cued Speech. Thus, the parents' use of signs (and Cued Speech) as communication support varied according to the abilities acquired from the courses. All the families received family-centered counselling and intervention from local Speech and Language Therapists approximately once a week during the first year after CI activation. According to the background questionnaire completed by the parents before implantation, there was only slight variation in family socioeconomic backgrounds as assessed by the level of parental education. Eleven of the mothers and ten of the fathers had tertiary educations (polytechnic degree or a lower/higher university degree), four of the mothers and fathers had secondary educations (professional or occupational education), and only one of the fathers had the lowest level of education (comprehensive school, i.e., primary and secondary school). During the first year after CI activation, the toddlers' hearing level gradually improved from pre-implant severe-profound 
Vocal Development with Two Cochlear Implants: Authors' accepted manuscript (AAM)

HL $(M=117, \mathrm{SD}=16.8)$ to mild HL $(M=32.1, S D=7.9)$. Hearing level was calculated as the pure tone average of the auditory thresholds at $0.5,1,2$ and $4 \mathrm{kHz}$ (if a response was not obtained at any frequency, a value of $130 \mathrm{~dB}$ HL was applied in the calculation; British Society of Audiology, 2011).

$<$ Table 1 approximately here >

The second group consisted of 15 TD infants with NH (eight boys and seven girls) who entered into this study at six months of age (hereafter referred to as the TD group). The TDgroup children were recruited through Child Welfare Clinics in Oulu ${ }^{1}$, Finland. The study was approved as ethically acceptable by the Ethics Committee of Social and Health Service, Oulu. Written consent was received from all the parents. According to the background questionnaire completed by the parents, the children came from monolingual Finnish-speaking homes, had normal developmental histories (i.e., no developmental delays, no impairments in vision or hearing nor motor disabilities ${ }^{1}$ ), and came from socioeconomic backgrounds (as assessed by the parental level of education) comparable to the bilateral CI group. Eleven of the mothers and ten of the fathers had a tertiary education and four of the mothers and five of the fathers had a secondary education. The children in the TD group had NH verified at the monthly appointments at Child Welfare Clinics ${ }^{1}$.

\section{Data collection}

Recordings. A prospective longitudinal research design was used in data collection. All infants and toddlers in the bilateral CI group were video and audio recorded with a wireless microphone (either Sony ECM-HW1T microphone and Sony Handycam DCR-SR90 or Canon WM-V1 microphone and Canon LEGRIA HF G25 video camera) placed near the infant-parent dyad. The recordings took place at the university hospitals once before implantation, and 1, 3, 6, 9, and 12 months after activation of the CIs according to the regular follow-up protocol. Two recordings were scheduled at the homes of the infants and toddlers 
Vocal Development with Two Cochlear Implants: Authors' accepted manuscript (AAM)

in order to make data collection possible (once for two different children; during summer vacations). Twenty-minute recordings consisted of semi-structured parent-child play sessions with a regular set of toys (i.e., LEGO® DUPLO $®$ farm set with toy animals, cars, books and puzzles). The infants of the TD group were video and audio recorded with a high-quality microphone (Sony ECM-HW1T microphone and Sony Handycam DCR-SR90) placed near the infant and the parent at the children's homes. These recordings also consisted of semistructured parent-child play sessions. The infants in the TD group were recorded at the chronological ages of 6,9 , and 12 months. These ages were chosen to match the amount of robust hearing of the infants and toddlers in the bilateral CI group (i.e. 6, 9, and 12 months of CI use). It was also expected that the TD group would produce varying types of vocalizations by 6 months of age (Koopmans-van Beinum \& van der Stelt, 1986; Oller, 1980; Roug et al., 1989; Stark, 1980). In both groups, the parents were instructed to interact with their child in their usual manner.

Transcription. A 20-minute sample was taken from the beginning of the recordings for broad phonetic transcription (for both groups) of the children's utterances by using the International Phonetic Alphabet (IPA Chart). An utterance was defined as a vocalization or group of vocalizations separated from other vocalizations by at least a one-second pause, an ingressive breath, or other person's speech (Ertmer \& Nathani Iyer, 2010; Stoel-Gammon, 1989). In addition, intonation or intuition of a natural pause were considered. Only speechlike vocalizations were considered as utterances, i.e., reflexive and vegetative sounds (e.g., hiccups, grunts, coughs, burps, snorts, crying) were disregarded. Trained transcribers transcribed all the vocalizations directly from the play session videotapes. If it was not possible to assign an IPA symbol to a segment according to broad transcription, the segment was marked with $\mathrm{V}$ or $\mathrm{C}$ and a written comment was added in the comment column. Two 
Vocal Development with Two Cochlear Implants: Authors' accepted manuscript (AAM)

transcribers transcribed $10 \%$ of the utterances to check the inter-rater reliability, which was found to be $88.7 \%$.

\section{Data analyses}

Classification of the utterances. The CSAEVD-R (Ertmer \& Nathani Iyer, 2010) was used for the classification of the vocalization types. For each sample, the first 50 utterances (or all utterances if $<50$ were available) adequate in intensity and without excessive background noise or talk-over were classified from each 20-minute session. Utterances with poor audio quality were disregarded from the analyses and replaced with the following consecutive utterance with appropriate audio quality. A written note on any quasivowels (short and quick vocalizations produced with closed mouth but with normal phonation; Oller, 2000, p. 158-160), slow vs. quick consonant-vowel or vowel-consonant transitions, and changes in the vowel quality was added in the comments column in order to refine the data analyses. The consonant-vowel or vowel-consonant transitions were perceptually judged as either slow or quick in order to verify canonical syllables. A canonical syllable was defined as having at least one fully resonant nucleus (i.e., vowel with an identifiable quality), one nonglottal margin (i.e., consonant other than glottal stop or glottal fricative), duration of syllable and formant transitions that (perceptually) fit with the range of mature syllable production, and normal phonation (Oller, 2000, p. 179-180). Changes in the vowel quality were also perceptually judged as either slow or quick in order to verify diphthongs vs. vowel glides.

A total of 5964 vocalizations were classified (3714 vocalizations for the bilateral CI group and 2250 vocalizations for the TD group) into one of the three levels, PC, BCS or AF of the CSAEVD-R according to the developmental level (Ertmer \& Nathani Iyer, 2010).

Classification was based on the transcripts, written comments and video and audio recordings. The CSAEVD-R analyzes speech-like utterances (protophones, the precursors of speech; Oller, 2000). Vocalization types that do not become more speech-like with age (e.g., 
Vocal Development with Two Cochlear Implants: Authors' accepted manuscript (AAM)

crying, fussiness, and vegetative sounds) are not analyzed to track vocal development (Ertmer \& Nathani Iyer, 2010). PC vocalizations are vocalizations that lack true vowel and consonant combinations with rapid transition between them. Examples of the PC vocalizations include quasivowels, isolated vowels, multiple vowel-like sounds (not diphthongs), or isolated consonants (e.g., [ə:], [ə:a:], [wawa], [m:]). BCS are vocalizations characterized by normal phonation, at least one consonant and one vowel combination with rapid transition between consonant and vowel. Examples of BCS are CV syllables and production of reduplicated and non-reduplicated babbling (e.g., [ka], [ta.ta], [m:ə.m:ə.m:ə], [ja.ja.ja], [ka.ta]). AF are vocalizations that have the characteristics of canonical syllables but have been reported to be more complex and later-emerging than CV syllables in TD infants and toddlers (Nathani et al., 2006). Examples of AF vocalizations include closed syllables (CVC, e.g., [həs:]), syllables starting with vowel (VC, VCV, e.g., [oup:]), consonant clusters (CCV, e.g., [tri]), and diphthongs (VV, e.g., [ai], [oi], [ou]). It is worth noting that the original five-level SAEVD-R has been found to be a reliable measure of vocal development when applied in a cross-sectional study of TD children with NH (Nathani et al., 2006). According to the researchers, the consolidation of the levels 1-3 into PC vocalizations has been possible, because PC vocalizations are routinely produced both by infants with $\mathrm{NH}$ and infants and toddlers who are deaf and distinguishing between these early emerging levels would not reveal true progress in auditory-guided vocal development (Ertmer et al., 2007; Nathani et al., 2006).

According to the principles used by Ertmer and colleagues (Ertmer \& Jung, 2012; Ertmer et al., 2013), the analysis was applied both for non-meaningful and meaningful utterances (if the child already produced meaningful utterances). The number of meaningful utterances was counted for each child and each data point according to the Vihman \& McCunes' (1994) criteria for words. For the bilateral CI group, all the utterances were non-meaningful before 
Vocal Development with Two Cochlear Implants: Authors' accepted manuscript (AAM)

implantation and during the first six months after CI activation. The mean proportion of meaningful utterances was $19.3 \%(S D=22.5 \%)$ at 9 months of CI use and $33.6 \%(S D=$ $26.9 \%$ ) at 12 months of CI use. The TD group produced meaningful utterances only at the chronological age of 12 months $(M=3 \% ; S D=7.0 \%)$.

Reliability. A random $10 \%$ sample of the utterances for both groups and from each data collection interval was reclassified to assess intra-classifier and inter-classifier reliability. Since the CSAEVD-R classification is ordered, the weighted kappa was calculated. The distance between PC and AF was weighted two times more than distance between the two immediately following levels (i.e., between PC and BCS or between BCS and AF). The weighted kappa was $0.99(\mathrm{z}=27.9, \mathrm{p}<0.001)$ for intra-rater (first author) agreement and 0.99 $(\mathrm{z}=27.7, \mathrm{p}<0.001)$ for inter-rater (first and second authors) agreement indicating almost perfect agreement (Landis \& Koch, 1977).

Statistical analysis. The data of the bilateral CI group were analyzed from the preimplantation interval, and 1, 3, 6, 9, and 12 months after CI activation. The TD group data were analyzed from 6, 9, and 12 months of chronological age. A maximum of 50 utterances was analyzed for each session. The number of child utterances that were classified at the PC, BCS, and AF levels was calculated for each follow-up session. The classified utterances were converted to percentages of PC, BCS and AF levels, because of differences in the total number of utterances per session. For the bilateral CI group, $11 \%$ of all sessions ( 10 sessions) had less than 50 utterances (range $=24-49$ utterances). Those sessions were from the following assessment intervals: pre-implantation, and 1 or 3 months after CI activation. For the TD group, all sessions had 50 utterances.

For the bilateral CI group, some data was missing, mainly because of the delay in the families' decision to participate in the study, families' inability to keep a given appointment, or because of an infant's and toddler's illness. For the purposes of the statistical modelling, 
Vocal Development with Two Cochlear Implants: Authors' accepted manuscript (AAM)

missing data was imputated for four infants and toddlers for the time-points of 3, 6, 9 and 12 months. Imputation was based on the data from the child's previous time-point. The consequence of the imputation decision was four imputations (one imputation per child; four different children). Pre-implantation data were not imputated, since there were not any previous data available. Independent samples $t$-test was used to analyze whether missing preimplantation data would bias the results of the vocal development follow-up. The analysis revealed no differences between the 'infants with missing pre-implantation data' vs. the 'infants with no missing data' for the PC vocalizations $(t=0.367, d f=11, p=0.720)$, BCS $(t$ $=1.597, d f=11, p=0.139)$ or for the $\mathrm{AF}(t=-1.111, d f=11, p=0.290)$ at one-month after CI activation. This indicates that the groups of infants and toddlers were not systematically selected, and the post-activation data could be interpreted as representative of vocal development of infants and toddlers with bilateral CIs. There were no missing data for the TD group.

Linear trends for vocal development of the bilateral CI group before and after CI activation with the time-points pre-implantation and 1, 3, 6, 9, and 12 months after CI activation were estimated by linear regression analysis, which was used to describe the behavior of the observed data without explaining it (Altman 1991, p. 212-220; Hess, Iyer, \& Malm, 2001). Linear trend estimation expresses data as a linear function of time and can also be used to determine the significance of differences in a set of data linked by a categorical factor. The trend is presented as change per month in percentage including analysis of $95 \%$ confidence limits (95\% CL). Comparable analysis was completed for the TD group with time-points of 6 , 9, and 12 months. Separate analyses were completed for PC, BCS and AF vocalizations. Linear trend analysis was also used to estimate the differences in the level of the trend lines between the bilateral CI and the TD groups. At first we ensured that the interaction term of time (with time-points 6, 9 and 12 months) and group (TD vs. bilateral CI) was insignificant 
Vocal Development with Two Cochlear Implants: Authors' accepted manuscript (AAM)

(PC vocalizations, beta $=0.91, t=0.53, p=0.59 ;$ BCS vocalizations, beta $=-0.007, t=-$ $0.007, p=0.99$; AF vocalizations, beta $=-0.91, t=-0.70, p=0.49)$. We then ensured that the residuals were normally distributed and the variance of the residuals was similar across the values. Effect sizes were determined by computing the value of the partial eta squared $\left(\eta P^{2}\right.$, Olejnik \& Algina, 2003), which indicates the ratio of variance accounted for by an effect and that effect plus its associated error variance. Throughout the Results section, effect sizes are reported using the set of conventions defined by Bakeman (2005): small equals 0.02, medium equals 0.13 , and large equals 0.26 .

\section{Results}

\section{Time-course of vocal development and group differences}

The first research question centered on investigating vocal development of infants and toddlers with bilateral CIs from pre-implant interval to 12 months after CI activation. The second research question centered on investigating vocal development of TD infants with $\mathrm{NH}$ during the time-period of 6 to 12 months. Linear trends with $95 \%$ confidence limits (CL) were estimated for vocal development for both groups separately.

The third research question centered on comparing vocal development of infants and toddlers with bilateral CIs to that of infants with NH and TD. For group comparison, the differences in the level of the linear trend lines with 95\% CL were estimated for the TD vs. the bilateral CI groups (time-points 6, 9 and 12 months). Separate analyses were completed for PC, BCS and AF vocalizations.

Precanonical Vocalizations (PC). Figure 1, topmost panel with open circles presents the mean percentage of the PC vocalizations of the bilateral CI group before implantation and 1, 3, 6, 9, and 12 months after CI activation. Figure 1, topmost panel with closed circles presents the mean percentage of PC vocalizations of the TD group for time-points 6, 9, and 12 months. From this figure, it can be seen that before implantation and during the first three months after 
Vocal Development with Two Cochlear Implants: Authors' accepted manuscript (AAM)

CI activation, the bilateral CI group produced mainly PC vocalizations $(M=90.8 \%, S D=6.6$ before implantation; $M=91.5 \%, S D=7.6$ one month after $\mathrm{CI}$ activation; $M=77.7 \%, S D=$ 20.4 three months after CI activation). The mean percentage of PC vocalizations decreased to $66.7 \%(S D=20.4)$ six months after CI activation, to $44.8 \%(\mathrm{SD}=23.3)$ at nine months postactivation, and to $25.6 \%$ at 12 months after CI activation. Figure 1 also shows that for the TD group, the mean percentage of the PC vocalizations decreased from $87.1 \%(\mathrm{SD}=15.4)$ at six months, to $74.3 \%(\mathrm{SD}=19.0)$ at nine months, and to $54.8 \%(\mathrm{SD}=21)$ at 12 months, respectively.

Linear regression analysis revealed that the early-emerging PC vocalizations decreased by $-5.36 \%$ in a month (with a large effect size) from pre-implantation to 12 months after CI activation for the bilateral CI group (Table 2). A quite similar trend was seen for the TD group. Their PC vocalizations decreased by $-5.37 \%$ in a month (with a large effect size) during the time-period of six to 12 months. Analysis of the group differences showed that the bilateral CI group produced a lower percentage of PC vocalizations than the TD group (Table 3; Figure 1). The estimated group difference was $25.18 \%$ over the period of 6 to 12 months of robust hearing, i.e., from 6 to 12 months of CI use for the bilateral CI group and from 6 to 12 months of age for the TD group.

$<$ Figure 1 approximately here>

$<$ Table 2 approximately here>

$<$ Table 3 approximately here>

Basic Canonical Syllables (BCS). The middle panel of Figure 1 shows that the proportions of BCS vocalizations were relatively low during the complete follow-up period from 6 to 12 months of robust hearing for both groups of children. For the bilateral CI group, the mean percentage of the BCS vocalizations increased from $5 \%(\mathrm{SD}=4)$ at pre-implant interval, to $12.6 \%(\mathrm{SD}=12.8)$ at six months after $\mathrm{CI}$ activation, to $19.4 \%(\mathrm{SD}=13.8)$ at nine 
Vocal Development with Two Cochlear Implants: Authors' accepted manuscript (AAM)

months after $\mathrm{CI}$ activation, and finally to $28.5 \%(\mathrm{SD}=16.5)$ at 12 months after CI activation (Figure 1, middle panel with open circles). Also, the TD group produced very few BCS vocalizations at six months of age $(\mathrm{M}=3.8, \mathrm{SD}=4.5)$ (Figure 1, middle panel with closed circles). The mean percentage of BCS vocalizations increased to $8.1 \%(\mathrm{SD}=6.9)$ at nine months, and to $18.4 \%(\mathrm{SD}=14.1)$ at 12 months of age.

When the developmental trends were analyzed with the linear regression analysis, the results revealed that the BCS vocalizations increased by $1.88 \% /$ month (with a large effect size) from pre-implant time-point to 12 months after CI activation for the bilateral CI group (Table 2, Figure 1). The TD group showed a quite similar trend with $2.44 \%$ /month increase in the proportion of BCS vocalizations during the time-period from 6 to 12 months. The group comparison revealed that the bilateral CI group produced a higher percentage of the BCS vocalizations than the TD group with an estimated group difference of $-9.56 \%$ (Table 3).

Advanced Forms (AF). Visual inspection of the lowest panel of Figure 1 reveals that the proportion of AF vocalizations steadily increased for both groups of children and that the proportion of AF vocalizations was higher than the proportion of BCS vocalizations. The mean percentage of the AF vocalizations for the bilateral CI group increased from a preimplant mean of $4.1 \%(\mathrm{SD}=5.4)$ to $12 \%(\mathrm{SD}=10.3)$ at three months, to $20.6 \%(\mathrm{SD}=11.8)$ at six months, and to $45.9 \%(\mathrm{SD}=19.6)$ at 12 months after CI activation (Figure 1, lowest panel) with open circles). For the TD group, an increase from $9.1 \%(\mathrm{SD}=11.8)$ at six months to $26.7 \%$ ( $\mathrm{SD}=14.5 .8$ ) at 12 months was noted (Figure 1 , lowest panel with closed circles). The linear regression analysis again revealed a quite similar trend for the two groups of infants and toddlers. For the bilateral CI group, the AF vocalizations increased by 3.49\%/month (with a large effect size) from pre-implant time-point to 12 months after CI activation (Table 2). For the TD group, the AF vocalizations increased by $2.94 \% /$ month. In close resemblance to the group differences regarding BCS vocalizations, the bilateral CI 
Vocal Development with Two Cochlear Implants: Authors' accepted manuscript (AAM)

group produced a higher percentage of the AF vocalizations than the TD group (Table 3). The estimated group difference was $-15.76 \%$ throughout the period.

In summary, the linear trends in the decrease of the PC vocalizations and in the increase of the BCS and AF vocalizations were quite similar for the bilateral CI and the TD groups (see Table 2). However, the bilateral CI group produced fewer PC vocalizations, but more BCS and AF vocalizations with comparable months of robust hearing.

\section{Discussion}

\section{The time-course of vocal development and group differences}

Precanonical Vocalizations. One of the first outcomes of the current study highlights the effect of profound HL on vocal development prior to implantation: the infants and toddlers in the bilateral CI group produced mainly the early-emerging PC vocalizations before CI activation. Because the mean chronological age at CI activation in the bilateral CI group was 13.3 months, they could have been expected to produce higher proportions of BCS and, possibly, some AF vocalizations at this age. The typical onset of babbling is reported to be between 6-10 months of age in infants with NH and TD (Oller, 2000, p. 179-180; Oller et al., 1999; Vihman, 1996, p. 109). The current study highlights that despite the era of newborn hearing screening, early HL identification and HA fitting, profound HL can still be considered as a risk factor for vocal development (e.g., Eilers \& Oller, 1994; Koopmans-van Beinum et al., 2001; Oller \& Eilers, 1988; Stoel-Gammon \& Otomo, 1986; von Hapsburg \& Davis, 2006). This information adds to the previous documentation of vocal development in infants and toddlers with severe-to-profound HL before implantation (Ertmer et al., 2007; Ertmer \& Jung, 2012; Ertmer et al., 2013; Schauwers et al., 2004; Schauwers et al., 2008).

The results of the current study are somewhat in contrast with the findings by Ertmer and colleagues (2007). They reported that three out of seven infants and toddlers in their study had already established BCS level (at $\geq 20 \%$ of the sample) before implant activation 
Vocal Development with Two Cochlear Implants: Authors' accepted manuscript (AAM)

(“establishment” criterion previously applied by Oller \& Eilers, 1988). It may well be that some toddlers with older age at CI activation (20, 24, and 36 months in Ertmer et al. study) might start to produce canonical syllables before implantation with continued HA use, possible residual hearing and intervention. However, vocal development would still be delayed compared to infants and toddlers with TD and NH.

The current study provided new information on precanonical vocalizations also for infants with TD and NH acquiring Finnish. In the only previously published study on Finnish infants, Liukkonen and Kunnari (2012) reported that $79.9 \%$ of the vocalizations of eight-month-old infants were precanonical. Thus, the proportion of the least mature and early-emerging PC vocalizations at nine months of age reported in the current study was in close resemblance to the previous findings on infants with TD and NH acquiring Finnish.

Basic Canonical Syllables and Advanced Forms. A second clear outcome of the current study is the evident advancement in the vocal development for the infants and toddlers with bilateral CIs after CI activation. The BCS vocalizations increased by $1.88 \% /$ month during the first year after CI activation and the BCS level was almost established nine months after CI activation (at $\geq 20 \%$ of the sample). The AF vocalizations increased by $3.49 \% /$ month during the first year of bilateral CI use and the level was established at six months after CI activation. If we consider the canonical BCS and AF levels together, over $20 \%$ of the sample was either BCS or AF vocalizations three months after CI activation. Thus, the results of the current study are in general agreement with previous reports on the advancement and rate of the vocal development in infants and toddlers with unilateral CI after CI activation (e.g., Ertmer \& Jung, 2012; Ertmer \& Mellon, 2001; Ertmer et al., 2007; Fagan, 2015; McCaffrey et al., 1999; Moore \& Bass-Ringdahl, 2002; Schauwers et al., 2004). Studies that have analyzed either onset of canonical babbling or proportions of BCS and AF vocalizations have 
Vocal Development with Two Cochlear Implants: Authors' accepted manuscript (AAM)

indicated clear advancements in vocal development starting from one to six months after CI activation.

Thirdly, the results on the increase in the proportions of the BCS and the AF vocalizations during the first year of bilateral CI use do not seem to lend support for a more-rapid-thantypical time-course of vocal development in the relatively young bilateral CI recipients of the current study compared to previous reports on older toddlers with unilateral CIs. The timecourse in the increase of the proportion of BCS and AF vocalizations after three months of CI use for the bilateral CI group of the current study was quite similar to the one reported in several previous studies on infants and toddlers with unilateral CIs (e.g., Ertmer \& Jung, 2012; Ertmer et al., 2013; Ertmer et al., 2007; Fagan, 2015; Schauwers et al., 2004).

Obviously, any conclusions have to be made cautiously, because the current study did not have matched groups of infants and toddlers with unilateral CI and bilateral CIs. The only comparison can be made with current literature. There are reports indicating that infants and toddlers with bilateral CIs use more vocalization to communicate, and use more audition when interacting vocally with an adult compared to infants and toddlers with unilateral CI (Tait et al., 2010). Thus, more research is needed e.g., on volubility, the amount of canonical syllables, the variety of syllable shapes and phoneme inventory (see e.g., Fagan, 2014, 2015; Schauwers et al., 2008), aspects of vocal development that might be more sensitive measures to track the developmental trends after bilateral implantation.

In addition to tracking the time-course of vocal development in BCS and AF vocalizations for the bilateral CI group, the current study revealed new results also on infants with TD. For the TD group, the BCS vocalizations increased somewhat between the ages 6 and 12 months and the BCS level was almost established at the age of 12 months (at $\geq 20 \%$ of the sample). The AF-level vocalizations also increased steadily and the AF level was established at 12 months of age. These findings highlight that the infants with $\mathrm{NH}$ who were acquiring Finnish 
Vocal Development with Two Cochlear Implants: Authors' accepted manuscript (AAM)

were already producing a variety of different vocalization types during the time-period that canonical vocalizations are typically produced (e.g., Oller, 2000, p. 179-180; Oller et al., 1999; Vihman, 1996, p. 109).

It is worth noting that the CSAEVD-R focuses on early phonetic and phonological development, since it analyzes speech-like utterances (Ertmer \& Jung, 2012; Ertmer et al., 2013). The analysis is applied both for non-meaningful and meaningful utterances. The increase of BCS and especially AF level vocalizations with complex syllable shapes between 9 to 12 months of robust hearing in both groups of children may in part resemble the syllable shape diversity of meaningful words (as also reported by Ertmer et al., 2013). Indeed, the toddlers in the bilateral CI group were attempting some meaningful words after 9 months of CI use (mean $19.3 \%$ at 9 months of CI use and $33.6 \%$ at 12 months of CI use). The same holds true for the TD group at the chronological age of 12 months (mean 3\%). Thus, the language-specific phonological features are obviously reflected in the toddlers' and infants' utterances. This fact further highlights the need for deeper analysis on the variety of syllable shapes and phoneme inventory.

Group differences. The current study showed clear differences between the infants and toddlers with bilateral CIs and infants with TD throughout the complete follow-up period. The bilateral CI group produced fewer PC vocalizations, but more BCS and AF vocalizations than the TD group with comparable months of robust hearing. As such, these findings are quite in line with those of Ertmer and Jung (2012) and Ertmer et al. (2013) who also found more rapid advancements in toddlers with CI than in TD infants with a comparable time of robust hearing. Thus, the current study supports the "Advanced Maturity" hypothesis proposed by Ertmer and colleagues to account for relatively rapid advancements following CI activation (Ertmer \& Jung, 2012; Ertmer et al., 2013). That is, the more advanced neurological, physiological, and motor, tactile and proprioceptive development of the bilateral 
Vocal Development with Two Cochlear Implants: Authors' accepted manuscript (AAM)

CI group could enable them to make rapid gains in vocal development, once robust hearing is introduced with the help of CI (see also e.g., Eilers \& Oller, 1994; Kuhl, 2004; Vihman, De Paolis, \& Keren-Portnoy, 2009).

The results of the current study, however, highlight that despite the rapid advancements after bilateral CI activation the infants and toddlers with profound HL lag behind their peers with $\mathrm{NH}$ in their vocal development. Namely, the bilateral CI group reached the mean proportion of $19.4 \%$ in the BCS vocalizations nine months after CI activation at the mean chronological age of 22.3 months (range 19-31). The TD group reached the mean proportion of $18.4 \%$ in the BCS vocalizations by the chronological age of 12 months. Furthermore, the bilateral CI group reached the level of $20.6 \%$ in the AF vocalizations six months after CI activation at the mean chronological age of 19.3 months (range 16-28). The TD group reached a comparable level at the chronological age of 12 months. Taken together, the bilateral CI group showed a mean delay of 7-9 months in their vocal development during the first year after CI activation compared to TD infants with NH. For some of the youngest toddlers with bilateral CIs there was a delay of only approximately three months, but for the oldest toddlers with bilateral CIs the delay could be approximately nine months.

\section{Cross-linguistic differences and considerations on the application of the CSAEVD-R}

\section{into Finnish}

Two main differences with English-based studies regarding the proportion of the AF vocalizations were noted, when the CSAEVD-R was used in the current study of infants and toddlers acquiring Finnish. First, the bilateral CI group produced a high proportion of AF vocalizations (established at $20 \%$ or greater) after only six months of robust listening experience with CIs. In contrast, English-acquiring CI infants and toddlers in Ertmer and Jung's (2012) study did not reach this level until nine months post activation. When discussing this difference, it is obviously important to notice that the mean age at CI 
Vocal Development with Two Cochlear Implants: Authors' accepted manuscript (AAM)

activation in Ertmer and Jung's study was considerably higher, there was a considerable variation in the age of $\mathrm{CI}$ activation $(M=20$; range 9-36 months), and a majority of the infants and toddlers were using a unilateral CI (10/13 participants). Since the two groups of young CI recipients were different from each other in many ways, any comparisons between the bilateral CI group of the current study and the CI group in Ertmer and Jung's study have to be considered cautiously.

A second difference regarding the proportion of AF vocalizations was, however, noted for the TD group. The proportion of AF vocalizations was higher at nine months $(M=17.6 \%)$ and 12 months $(M=26.7 \%)$ of age also for the infants and toddlers with NH acquiring Finnish than for infants and toddlers with $\mathrm{NH}$ acquiring English $(M=3.5 \%$ and $M=4.1 \%$, respectively, see Ertmer \& Jung, 2012). Thus, the high proportion of the AF vocalizations in the current study clearly deserves a closer look. In the CSAEVD-R (Ertmer \& Nathani Iyer, 2010), diphthongs are classified into AF vocalizations based on their later emergence in the productions of infants and toddlers with NH acquiring American English (Nathani et al., 2006). In General American English, there are only three or four phonemic diphthongs (Bosma Smit, 2007) as opposed to 18 in Finnish (Suomi et al., 2006). This is a clear crosslinguistic difference, which may be reflected in the different results between the current study and the study by Ertmer and Jung (2012); two studies that otherwise followed quite similar study designs.

Previous reports on the syllable structures attempted by infants and toddlers with $\mathrm{NH}$ acquiring Finnish offer another interesting platform for discussion of cross-linguistic differences between infants and toddlers acquiring General American English and Finnish. There is ample evidence that Finnish infants and toddlers produce a very high proportion of VV syllable structures during the period of the first 50 words; these may be either meaningful or non-meaningful utterances (Kunnari, 2000, p. 57; Savinainen-Makkonen, 2000; Vihman \& 
Vocal Development with Two Cochlear Implants: Authors' accepted manuscript (AAM)

Velleman, 2000). This might also be reflected in the different proportions of AF vocalizations found in the current study. For the infants and toddlers of the current study, VV syllable structure and diphthongs were often found in the utterances, e.g. [æi.je], a non-meaningful vocalization with (.) marking the syllable break or [ei] 'no', a meaningful utterance. The aforementioned findings lend clear support to the emerging language-specific processes during vocal development for both infants and toddlers with TD as well as for infants and toddlers with bilateral CIs (Boysson-Bardies \& Vihman, 1991; DePaolis, Vihman, \& Kunnari, 2008; Kunnari, 2000; Saaristo-Helin, Kunnari, \& Savinainen-Makkonen, 2011). The findings highlight the need for more detailed analyses of the vocalizations of infants and toddlers with bilateral CIs and also with TD who are acquiring Finnish during the prelinguistic stage. Such analyses would be foundational for the development of a Finnish version of the CSAEVD-R.

\section{Clinical implications}

The results of the current study raise several issues to be considered in early intervention. First, despite HL diagnosis through newborn hearing screening and early HA fitting, delays are to be expected in the vocal development of infants with severe-to-profound HL before implantation. Second, significant increases in the proportion of canonical syllables and more advanced forms may be expected by three months with robust listening experience with bilateral CIs. Progress toward more complex utterances may be expected between six and twelve months after activation for Finnish-acquiring infants and toddlers with bilateral CIs. If the proportion of the canonical syllables with complex syllable shapes is observed to be very low or even non-existent between six and 12 months post-activation, referral to more comprehensive assessment of CI functioning and infants' and toddlers' development should be considered. Third, vocal development in infants and toddlers with early HA fitting and even early bilateral CI use may be delayed at least during the first year after CI activation 
Vocal Development with Two Cochlear Implants: Authors' accepted manuscript (AAM)

compared to infants and toddlers with $\mathrm{NH}$. This information is important for the parents of infants and toddlers with bilateral CIs. Parental expectations of spoken language development soon after bilateral CI activation are often high. Parents are often aware of the better sound localization and speech perception ability in background noise reported for children with bilateral CIs (Johnston et al., 2009). Thus, there are often discussions on whether greater benefits might be gained also in the acquisition of mature speech patterns with bilateral CI use. Knowledge of the levels and time-course of vocal development after bilateral implantation may help parents recognize progress in auditory-guided speech development even before the emergence and the use of the spoken words in communication on a regular basis.

\section{Limitations and future directions}

Several limitations need to be considered when interpreting the results of the current study. It would have been advantageous to have pre-implantation data from all the infants and toddlers in the bilateral CI group. Unfortunately, this was not possible because of some delays in the parents' decision to participate in the study or inability to keep the appointment (due to e.g., infants and toddlers' illness). Such aspects are hard to avoid, especially in a nationwide, multi-center study with long traveling distances. Another limitation was the three-month time-interval of the data collection. More frequent data collection would have provided support to more precise analyses for the sequence and rate of (prelinguistic) vocal development. However, a pre-decided protocol was necessary in order to control the data collection in a multi-center clinical study design. Further studies are needed to determine whether the reported benefits of bilateral implantation on children's speech perception abilities in noise (Johnston et al., 2009) can be observed in the vocal development of infants and toddlers with bilateral CIs, e.g., in productions of more complex syllable shapes or 
Vocal Development with Two Cochlear Implants: Authors' accepted manuscript (AAM)

consonant variegations. More detailed analysis might reveal additional information on the possible positive effects of bilateral implantation for vocal development.

In conclusion, the findings of this study showed that the vocal development of the infants and the toddlers with profound HL is delayed before CI activation. Once they receive bilateral CIs, they make rapid advancements in this foundational linguistic phenomenon. However, their vocal development seems to remain delayed at least during the first year after bilateral CI activation compared to the well-documented milestones reached by infants with $\mathrm{NH}$ and TD.

\section{Acknowledgements}

The current study was supported by the Academy of Finland. The authors are especially grateful for the support of multiple colleagues at all five university hospitals in Finland (Helsinki, Kuopio, Oulu, Tampere and Turku) for recruiting children of this national multicenter research, for their help in assessing the children and for allowing the authors to use their facilities. These valued individuals and departments are Heikki Löppönen, MD, Professor from the University of Eastern Finland, Institute of Clinical Medicine, Department of Otorhinolaryngology and Kuopio University Hospital, Department of Otorhinolaryngology, Finland; Eila Lonka, PhD, SLT, Research Unit of Logopedics, University of Oulu, Finland; Antti A. Aarnisalo, MD, Adjunct Professor, University of Helsinki and Helsinki University Hospital, Department of Otorhinolaryngology-Head and Neck Surgery, Finland; Nonna Virokannas, MA, SLT, Helsinki University Hospital, Department of Otorhinolaryngology-Head and Neck Surgery, Finland; Ville Sivonen, PhD (Tech.), Helsinki University Hospital, Department of Otorhinolaryngology-Head and Neck Surgery, Finland; Antti Hyvärinen, MD, Kuopio University Hospital, Department of Otorhinolaryngology, Finland; Teija Tsupari, MA, SLT, Kuopio University Hospital, Department of Otorhinolaryngology, Finland; Aarno Dietz, MD, PhD, Kuopio University 
Vocal Development with Two Cochlear Implants: Authors' accepted manuscript (AAM)

Hospital, Department of Otorhinolaryngology, Finland; Jaakko Laitakari, MD, PhD, Oulu

University Hospital, Department of Otorhinolaryngology-Head and Neck Surgery, Finland;

Tanja Tennilä, MA, SLT, Oulu University Hospital, Department of Otorhinolaryngology-

Head and Neck Surgery, Finland; Jaakko Salonen, MD, PhD, Turku University Hospital,

Department of Otorhinolaryngology-Head and Neck Surgery, Finland; Satu Rimmanen, MA,

SLT, Turku University Hospital, Department of Otorhinolaryngology-Head and Neck

Surgery, Finland; Sari Mykkänen, MD, Tampere University Hospital, Department of

Otorhinolaryngology, Finland; Sari Vikman, MA, SLT, Tampere University Hospital,

Department of Otorhinolaryngology, Finland; Anna-Kaisa Tolonen, MA, SLT, PhD student,

Research Unit of Logopedics, University of Oulu, Finland; Krista Wallenius, MA, SLT, PhD

Student, Research Unit of Logopedics, University of Oulu, Finland. We also sincerely thank all the families and children who participate in this ongoing study.

\section{Footnote}

${ }^{1}$ The Health Care Act of Finland (1326/2010 and 338/2011) requires each local authority to provide primary health care for all its inhabitants at Health Centers, and for infants at Child Welfare Clinics. According to the present practices of Child Welfare Clinics, each child must be provided with nine comprehensive health surveillances including developmental tests and screening of vision and hearing by child health nurses and general practitioners during their first year of life. 


\section{References}

Altman, D. G. (1991). Practical Statistics for Medical Research. London: Chapman and Hall.

Bakeman, R. (2005). Recommended effect size statistic for repeated measures design. Behavior Research Methods, 37, 379-384. https://doi.org/10.3758/BF03192707

Bosma Smit, A. (2007). General American English speech acquisition. In S. McLeod (ed.), The international guide to speech acquisition (p. 128-147). Clifton Park, NY: Thomson Delmar Learning.

Boysson-Bardies de, B. \& Vihman, M. M. (1999). Adaptation to Language: Evidence from babbling and first words in four languages. Language, 67, 297-319. https://doi.org/10.1353/lan.1991.0045

Davis, B. L., Morrison, H. M., von Hapsburg, D., \& Warner Czyz, A. D. (2005). Early vocal patterns in infants with varied hearing levels. The Volta Review, 105, 7-27. https://search.proquest.com/docview/199058847?accountid=13031

DePaolis, R. A., Vihman, M. M., \& Kunnari, S. (2008). Prosody in production at the onset of word use: a cross-linguistic study. Journal of Phonetics, 36, 406-422. https://doi.org/10.1016/j.wocn.2008.01.003

Eilers, R. E. \& Oller, D. K. (1994). Infant vocalizations and the early diagnosis of severe hearing impairment. Journal of Pediatrics, 124, 199-203. https://doi.org/10.1016/S0022-3476(94)70303-5

Ertmer, D. J. \& Jung, J. (2012). Prelinguistic vocal development in young cochlear implant recipients and typically developing infants: Year 1 of robust hearing experience. Journal of Deaf Studies and Deaf Education, 17, 116-132. https://doi.org/10.1093/deafed/enr021

Ertmer, D. J., Jung, J., \& True Kloiber, D. (2013). Beginning to talk like an adult: Increases in speech-like utterances in young cochlear implant recipients and typically developing 
Vocal Development with Two Cochlear Implants: Authors' accepted manuscript (AAM)

children. American Journal of Speech-Language Pathology, 22, 591-603. https://doi.org/10.1044/1058-0360(2013/12-0058)

Ertmer, D. J. \& Mellon, J. A. (2001). Beginning to talk at 20 months: Early vocal development in a young cochlear implant recipient. Journal of Speech, Language, and Hearing Research, 44, 192-206. https://doi.org/10.1044/1092-4388(2001/017)

Ertmer, D. J., \& Nathani Iyer, S. (2010). Prelinguistic vocalizations in infants and toddlers with hearing loss: Identifying and stimulating auditory-guided speech development. In M. Marschark \& P. Spencer (Eds.), The Oxford University handbook of deaf studies, language, and education (Vol. 2, pp. 360-375). New York, NY: Oxford University Press.

Ertmer, D. J., Young, N. M., \& Nathani, S. (2007). Profiles of vocal development in young cochlear implant recipients. Journal of Speech, Language, and Hearing Research, 50, 393-407. https://doi.org/10.1044/0161-1461(2002/016)

Fagan, M. K. (2014). Frequency of vocalization before and after cochlear implantation: Dynamic effect of auditory feedback on infant behavior. Journal of Experimental Child Psychology, 126, 328-338. https://doi.org/10.1016/j.jecp.2014.05.005

Fagan, M. (2015). Why repetition? Repetitive babbling, auditory feedback, and cochlear implantation. Journal of Experimental Child Psychology, 137, 125-136. https://doi.org/10.1016/j.jecp.2015.04.005

von Hapsburg, D. \& Davis, B. (2006). Auditory sensitivity and the prelinguistic vocalizations of early-amplified infants. Journal of Speech, Language, and Hearing Research, 49, 809-822. https://doi.org/10.1044/1092-4388(2006/057)

Hess, A., Iyer, H., \& Malm, W. (2001). Linear trend analysis: a comparison of methods. Atmospheric Environment, 35, 5211-5222. https://doi.org/10.1016/S13522310(01)00342-9 
Vocal Development with Two Cochlear Implants: Authors' accepted manuscript (AAM)

Häkkinen, K. (1978). Eräistä suomen kielen äännerakenteen luonteenomaisista piirteistä ja niiden taustasta. Unpublished licentiate thesis. Department of Finnish and General Linguistics, University of Turku. [On some characteristics of the Finnish phoneme system and their origins]

IPA Chart, Retrieved from http://www.internationalphoneticassociation.org/content/ipa-chart, available under a Creative Commons Attribution-Sharealike 3.0 Unported License. Copyright () 2005 International Phonetic Association.

Johnston, J. C., Durieux-Smith, A., Angus, D., O’Connor, A., \& Fitzpatrick, E. (2009). Bilateral paediatric cochlear implants: A critical review. International Journal of Audiology, 48, 601-617. https://doi.org/10.1080/14992020802665967

Kern, S., \& Davis, B. L. (2009). Emergent complexity in early vocal acquisition: Crosslinguistic comparisons of canonical babbling. Approaches to Phonological Complexity, $353-375$.

Koopmans-van Beinum, F. J., Clement, C. J., \& van den Dikkenberg-Pot, I. (2001). Babbling and the lack of auditory speech perception: a matter of coordination. Developmental Science, 4, 61-70. https://doi.org/10.1111/1467-7687.00149

Koopmans-van Beinum, F.J. \& van der Stelt, J.M. (1986). Early stages in the development of speech movements. In B. Lindblom, \& R. Zetterström (eds.), Precursors of early speech (p. 37-50). New York: Stockton.

Kuhl, P. K. (2004). Early language acquisition: Cracking the speech code. Nature, 5, 831843. https://doi.org/10.1038/nrn1533

Kunnari, S. (2000). Characteristics of early lexical and phonological development in children acquiring Finnish. Acta Universitatis Ouluensis, Oulu University Press. Doctoral dissertation. 
Vocal Development with Two Cochlear Implants: Authors' accepted manuscript (AAM)

Kunnari, S. \& Savinainen-Makkonen, T. (2007). Finnish speech acquisition. In S. McLeod (ed.), The international guide to speech acquisition (p. 351-363). Clifton Park, NY: Thomson Delmar Learning.

Landis, J. R. \& Koch, G. G. (1977). The measurement of observer agreement for categorical data. Biometrics, 33, 159-174. https://doi.org/10.2307/2529310

Levitt, A. G., \& Aydelott Utman, J. G. (1992). From babbling towards the sound systems of English and French: A longitudinal two-case study. Journal of Child Language, 19, 1949. https://doi.org/10.1017/S0305000900013611

Liukkonen, H. \& Kunnari, S. (2012). Ääntelyn kehittyneisyys 8 kuukauden iässä ja sen yhteys varhaisen tuottavan sanaston kokoon. Puhe ja kieli, 32, 3-15. [Complexity of vocal production at the age of 8 months and its relationship to the size of early productive vocabulary. Speech and language, 32, 3-15.]

McCaffrey, H. A., Davis, B. L., MacNeilage, P. F., \& Von Hapsburg, D. (1999). Multichannel cochlear implantation and the organization of early speech. Volta Review, $101,5-28$.

Moeller, M. P., Hoover, B., Putman, C., Arbataitis, K., Bohnenkamp, G., Peterson, B., Lewis, D., ... Stelmachowicz, P. (2007). Vocalizations of infants with hearing loss compared with infants with normal hearing: Part II - Transition to Words. Ear and Hearing, 28, 628-642. https://doi.org/10.1097/AUD.0b013e31812564ab

Moore, J. A. \& Bass-Ringdahl, S. (2002). Role of infant vocal development in candidacy for and efficacy of cochlear implantation. The Annals of Otology, Rhinology \& Laryngology, 189, 52-55. https://doi.org/10.1177/00034894021110S511

Moreno-Torres, I. (2014). The emergence of productive speech and language in Spanishlearning paediatric cochlear implant users. Journal of Child Language, 41, 575-599. https://doi.org/10.1017/S0305000913000056 
Vocal Development with Two Cochlear Implants: Authors' accepted manuscript (AAM)

Nathani, S., Ertmer, D. J., \& Stark, R. J. (2006). Assessing vocal development in infants and toddlers. Clinical Linguistics \& Phonetics, 20, 351-369. https://doi.org/10.1080/02699200500211451

Nathani, S., Oller, D. K., \& Neal, A. R. (2007). On the robustness of vocal development: An examination of infants with moderate-to-severe hearing loss and additional risk factors. Journal of Speech, Language, and Hearing Research, 50, 1425-1444. https://doi.org/10.1044/1092-4388(2007/099)

Olejnik, S. \& Algina, J. (2003). Generalized eta and omega squared statistics: Measures of effect size for some common research designs. Psychological Methods, 8, 434-447. https://doi.org/10.1037/1082-989X.8.4.434

Oller, D. K. (1980). The emergence of the sounds of speech in infancy. In G.H. YeniKomshian, J. F. Kavanagh, \& C. A. Ferguson (eds.), Child phonology. Vol. 1, (p. 93112). New York: Academic Press.

Oller, D. K. (2000). The emergence of the speech capacity. Mahwah, NJ: Erlbaum.

Oller, D. K. \& Eilers, R. E. (1988). The role of audition in infant babbling. Child Development, 59, 441-444.

Oller, D. K., Eilers, R. E., Neal, A. R., \& Schwartz, H. K. (1999). Precursors to speech in infancy: The prediction of speech and language disorders. Journal of Communication Disorders, 32, 223-245. https://doi.org/10.2307/1130323

Roug, L., Landberg, I., \& Lundberg, L. -J. (1989). Phonetic development in early infancy: A study of four Swedish children during the first eighteen months of life. Journal of Child Language, 16, 19-40. https://doi.org/10.1017/S0305000900013416

Saaristo-Helin, K., Kunnari, S., \& Savinainen-Makkonen, T. (2011). Phonological development in children learning Finnish: A review. First Language, 31, 342-363. https://doi.org/10.1177/0142723710396793 
Vocal Development with Two Cochlear Implants: Authors' accepted manuscript (AAM)

Savinainen-Makkonen, T. (2000). Word-initial consonant omissions: A developmental process in children learning Finnish. First Language, 20, 161-185. https://doi.org/10.1177/014272370002005903

Schauwers, K., Gillis, S., Daemers, K., De Beukelaer, C., \& Govaerts, P.J. (2004). Cochlear implantation between 5 and 20 months of age: The onset of babbling and the audiologic outcome. Otology \& Neurotology, 25, 253-270.

Schauwers, K., Gillis, S., \& Govaerts, P.J. (2008). The characteristics of prelexical babbling after cochlear implantation between 5 and 20 months of age. Ear and Hearing, 29, 627637. https://doi.org/10.1097/AUD.0b013e318174f03c

Stark, R. E. (1980). Stages of speech development in the first year of life. In G. H. YeniKomshian, J. F. Kavanagh, \& C. A. Ferguson (Eds.), Child phonology. Vol. 1, (p. 7392). New York: Academic Press.

Stoel-Gammon, C. (1985). Phonetic inventories, 15-24 months: A longitudinal study. Journal of Speech, Language, and Hearing Research, 28, 505-512. https://doi.org/10.1044/jshr.2804.505

Stoel-Gammon, C. (1989). Prespeech and early speech development of two late talkers. First Language, 9, 207-224. https://doi.org/10.1177/014272378900900607

Stoel-Gammon, C. \& Otomo, K. (1986). Babbling development of hearing-impaired and normally hearing subjects. Journal of Speech and Hearing Disorders, 51, 33-41. https://doi.org/10.1044/jshd.5101.33

Suomi, K., Toivanen, J., \& Ylitalo, R. (2006). Fonetiikan ja äänneopin perusteet. Helsinki: Gaudeamus. [The fundamentals of phonetics and phonology]

Tait, M., Nikolopoulos, T.P., De Raeve, L., Johnson, S., Datta, G., Karltorp, E., Ostlund, E., ... Frijns, J.H.M. (2010). Bilateral versus unilateral cochlear implantation in young 
Vocal Development with Two Cochlear Implants: Authors' accepted manuscript (AAM)

children. International Journal of Pediatric Otorhinolaryngology, 74, 206-211.

https://doi.org/10.1016/j.ijporl.2009.11.015

Vihman, M. M. (1996). Phonological development: the origins of language in the child. Cambridge: Blackwell.

Vihman, M., DePaolis, R. A., \& Keren-Portnoy, T. (2009). A dynamic systems approach to babbling and words. In E. L. Bavin (Ed.), The Cambridge Handbook of Child Language (pp. 163-184). (Cambridge Handbooks in Linguistics). Cambridge: Cambridge University Press.

Vihman, M. M., Macken, M. A., Miller, R., Simmons, H., \& Miller, J. (1985). From babbling to speech: A re-assessment of the continuity issue. Language, 61, 397-445. https://doi.org/10.2307/414151

Vihman, M. M. \& McCune, L. (1994). When is a word a word? Journal of Child Language, 21, 517-542. https://doi.org/10.1017/S0305000900009442

Vihman, M. M. \& Velleman, S. L. (2000). The construction of a first phonology. Phonetica, 57, 255-266. https://doi.org/10.1159/000028478

WHO, (2015). World Health Organization, WHO. Grades of hearing impairment. Retrieved from http://www.who.int/pbd/deafness/hearing_impairment_grades/en/ 
Vocal Development with Two Cochlear Implants: Authors' accepted manuscript (AAM)

\section{Figure captions}

Figure 1. Mean percentages of the Precanonical Vocalizations (PC; topmost panel), Basic Canonical Syllables (BCS; middle panel) and Advanced Forms (AF; lowest panel). The open circles represent infants and toddlers with bilateral cochlear implants $(\mathrm{CI})$ and the closed circles represent infants with normal hearing and typical development (TD). The number of infants and toddlers in the groups was as follows: 1) bilateral CI group; 9 infants at pre-implantation interval, 13 toddlers at 1 and 3 months after $\mathrm{CI}$ activation, and 15 toddlers at 6, 9 and 12 months after CI activation, 2) TD group; 15 infants at each time-point. The error bars represent $95 \%$ confidence limit (CL). 
Vocal Development with Two Cochlear Implants: Authors' accepted manuscript (AAM)

Table 1

Information on gender, etiology, age at first implant activation (in months), cochlear implant (CI) details, and the communication mode the parents used with their infants and toddlers in the bilateral CI group.

\begin{tabular}{llllllllc}
\hline ID & Gender & Etiology & Implantation & Activation & Implants & Processors & Strategy & Parents' communication mode \\
\hline ID1 & M & GJB2 & 11 & 14 & NucleusCI24RE & CP810 & ACE & Speech and signs \\
ID2 & M & unknown & 11 & $12^{2}$ & NucleusCI513 & CP810 & ACE & Speech and signs \\
ID3 & M & unknown & 16 & $18^{3}$ & NucleusCI422 & CP810 & ACE & Speech and signs \\
ID4 & M & GJB2 & 14 & 15 & NucleusCI24RE & CP810 & ACE & Speech and signs \\
ID5 & M & unknown & 11 & 12 & NucleusCI513 & CP810 & ACE & Speech and signs \\
ID6 & M & GJB2 & 11 & 12 & NucleusCI422 & CP810 & ACE & Speech and signs \& Cued speech \\
ID7 & M & GJB2 & 11 & 12 & NucleusCI24RE & CP810 & ACE & Speech and signs \\
ID8 & F & GJB2 & 13 & 14 & NucleusCI24RE & CP810 & ACE & Speech and signs \\
ID9 & F & GJB2 & 10 & 11 & NucleusCI24RE & CP810 & ACE & Speech and signs \& Cued speech \\
ID10 & M & unknown & 10 & 11 & NucleusCI422 & CP810 & ACE & Speech and signs \\
ID11 & F & GJB2 & 12 & 13 & NucleusCI422 & CP810 & ACE & Speech and signs \\
ID12 & F & GJB2 & 9 & 10 & NucleusCI422 & CP810 & ACE & Speech and signs \\
\hline
\end{tabular}


Vocal Development with Two Cochlear Implants: Authors' accepted manuscript (AAM)

\begin{tabular}{|c|c|c|c|c|c|c|c|c|}
\hline ID13 & $\mathrm{M}$ & GJB2 & 10 & 11 & NucleusCI24RE & CP810 & $\mathrm{ACE}$ & Speech and signs \\
\hline ID14 & M & GJB2 & 21 & 22 & NucleusCI422 & CP810 & $\mathrm{ACE}$ & Speech and signs \\
\hline ID15 & $\mathrm{F}$ & GJB2 & 11 & $12^{4}$ & Nucleus412 & CP810 & $\mathrm{ACE}$ & Speech and signs \\
\hline \multicolumn{9}{|c|}{ NucleusCI24RE ${ }^{5}$} \\
\hline
\end{tabular}

Note. ${ }^{1}$ Mutation in the GJB2 (Connexin26) gene; ${ }^{2}$ Second CI activation at 22 months of age (first CI used for 10 months); ${ }^{3}$ Second implant activation at 23 months of age (first CI used for 4 months); ${ }^{4}$ Second implant activation at 17 months of age (first CI used for 5 months); ${ }^{5}$ Model of the second CI. 
Vocal Development with Two Cochlear Implants: Authors' accepted manuscript (AAM)

Table 2

Linear regression analysis for the Precanonical Vocalizations (PC), Basic Canonical Syllables (BCS) and Advanced Forms vocalizations (AF) for infants and toddlers with bilateral cochlear implants (bilateral CI group) including time-points before implantation, and 1, 3, 6, 9, and 12 months after CI activation, and for infants with typical development and normal hearing (TD group) at 6, 9 and 12 months.

\begin{tabular}{lll}
\hline Change per & $95 \%$ CL(lower & Partial Eta \\
month\%(SE) & bound; upper & Squared $^{1}$ \\
& bound) & \\
\hline
\end{tabular}

Bilateral CI group (pre-implant, 1, 3, 6, 9, 12 months)

\begin{tabular}{llll}
\hline PC vocalizations & $-5.36(0.49)$ & $-6.25 ;-4.47$ & 0.64 \\
BCS vocalizations & $1.88(0.29)$ & $1.30 ; 2.46$ & 0.34 \\
AF vocalizations & $3.49(0.32)$ & $2.86 ; 4.13$ & 0.59 \\
\hline & TD group $(6,9,12$ months $)$ & \\
\hline PC vocalizations & $-5.37(1.13)$ & $-7.65 ;-3.11$ & 0.35 \\
BCS vocalizations & $2.44(0.58)$ & $1.28 ; 3.60$ & 0.30 \\
AF vocalizations & $2.94(0.87)$ & $1.16 ; 4.70$ & 0.21 \\
\hline
\end{tabular}

Note. $S E=$ standard error; $C L=$ confidence limit $;{ }^{1}$ Partial Eta Squared effect size (small equals 0.02 , medium equals 0.13 , and large equals 0.26 ). 
Vocal Development with Two Cochlear Implants: Authors' accepted manuscript (AAM)

Table 3

Estimated group differences in Precanonical Vocalizations (PC), Basic Canonical Syllables (BCS) and Advanced Forms vocalizations $(A F)$ for infants with typical development and normal hearing (TD group) and for infants and toddlers with bilateral cochlear implants (bilateral CI group) with time-points 6, 9, and 12 months.

\begin{tabular}{llll}
\hline & $\%(S E)$ & $\begin{array}{l}95 \% \text { CL }(\text { lower } \\
\text { bound; upper } \\
\text { bound })\end{array}$ & $\begin{array}{l}\text { Partial Eta } \\
\text { Squared }^{1}\end{array}$ \\
\hline PC vocalizations & & \\
\hline $\begin{array}{l}\text { Difference between } \\
\text { groups (TD vs. CI) }\end{array}$ & $25.18(4.19)$ & $16.85 ; 33.51$ & 0.35 \\
\hline BCS vocalizations & & & \\
\hline $\begin{array}{l}\text { Difference between } \\
\text { groups (TD vs. CI) }\end{array}$ & $-9.56(2.49)$ & $-14.51 ;-4.60$ & 0.16 \\
\hline AF vocalizations & & & \\
\hline $\begin{array}{l}\text { Difference between } \\
\text { groups (TD vs. CI) }\end{array}$ & $-15.76(3.17)$ & $-22.08 ;-9.45$ & 0.24
\end{tabular}

Note. $S E=$ standard error; $C L=$ confidence limit; ${ }^{1}$ Partial Eta Squared effect size (small equals 0.02 , medium equals 0.13 , and large equals 0.26 ). 